\title{
DESAFÍOS ÉTICOS DE LA INVESTIGACIÓN BIOMÉDICA EN TIEMPOS DE COVID-19: PANORAMA EN ARGENTINA
}

\author{
DESAFIOS ÉTICOS DA PESQUISA BIOMÉDICA NA ÉPOCA DE COVID-19: VISÃO \\ GERAL NA ARGENTINA
}

ETHICAL CHALLENGES OF BIOMEDICAL RESEARCH IN TIMES OF COVID-19: OUTLOOK IN ARGENTINA

DOI: $10.22481 /$ rbba.v1i02.7797

\begin{abstract}
Alejandro Raúl Trombert
Universidad Nacional del Litoral, Santa Fe, Argentina

ORCID: https://orcid.org/0000-0002-0658-9448

Endereço eletrônico: atrombert@ fbcb.unl.edu.ar

María Carolina Caputto

Universidad Nacional del Litoral, Santa Fe, Argentina

ORCID: http://orcid.org/0000-0001-7703-1970

Endereço eletrônico: carocaputto@ hotmail.com
\end{abstract}

\section{RESUMEN}

Luego de una breve introducción sobre la pandemia de COVID19, el presente artículo realiza un acotado y conveniente recorrido por la normativa y principales documentos internacionales referidos a las particularidades de la investigación con seres humanos en el contexto de emergencias sanitarias. Seguidamente, con referencia a la Argentina, cuestiona la falta de regulación adecuada, mencionando las normas existentes a nivel nacional que se aplican a los ensayos clínicos, destaca la creación de un Comité Nacional específico, tras lo cual persigue reflexionar acerca de la respuesta dada en el país frente a la actual pandemia sólo en lo que atañe a investigación, analizando y comentando -sucintamente pero no por ello sin espíritu crítico- las coincidencias, los aciertos y deficiencias de la Resolución $N^{\circ}$ 908/20 emitida por el Ministerio de Salud de la Nación, particularmente sobre las pautas éticas que impone a las investigaciones desarrolladas en el país en durante la emergencia. 
Palabras clave: COVID-19; Ética de la investigación con seres humanos; Investigación biomédica; Investigación en brotes epidémicos; Revisión ética acelerada; Pandemia.

\title{
RESUMO
}

Após uma breve introdução sobre a pandemia COVID-19, este artigo faz um limitado e conveniente passeio pelos regulamentos $\mathrm{y}$ principais documentos internacionais no que concerne às particularidades da pesquisa com seres humanos no contexto de emergências sanitárias. A seguir, com referência à Argentina, questiona a falta de regulamentação adequada, mencionando as normas existentes a nível nacional que se aplicam aos ensaios clínicos, destaca a criação de um Comitê Nacional específico, após a qual procura refletir sobre a resposta dada no país enfrentando a atual pandemia apenas no que diz respeito à pesquisa, analisando e comentando - de modo sucinto, mais não por iso sem espírito crítico- as coincidências, os acertos y deficiências da Resolução $N^{\circ}$ 908/20 do Ministério da Saúde nacional, em particular sobre as diretrizes éticas que impõe às pesquisas desenvolvidas no país durante a emergência.

Palavras-chave: COVID-19; Ética na investigação com seres humanos; Pesquisa biomédica; Pesquisa em surtos epidêmicos; Revisão ética acelerada; Pandemia.

\begin{abstract}
After a brief introduction about the COVID-19 pandemic, this article makes a detailed and convenient review of the regulation and the main international documents that refer to the distinctive issues of research with human beings in the context of health emergencies. Then, with reference to Argentina, it addresses the lack of adequate regulation, mentioning the existing regulations at the national level that apply to clinical trials and highlighting the creation of a specific National Committee. Finally the contribution pursues to reflect on the response given in the country to the current pandemic with regard to research specifically, analyzing and commenting - briefly but critically-, the coincidences, successes and deficiencies of Resolution $\mathrm{N}^{\circ}$ 908/20, issued by the Ministry of Health of the Nation, particularly on the ethical guidelines that regulate the research carried out in the country during the emergency.
\end{abstract}

Keywords: COVID-19; Ethics of Research with Human Beings; Biomedical research; Research in infectious disease outbreaks; Accelerated ethics review; Pandemic. 


\section{INTRODUCCIÓN}

La COVID-19 (acrónimo del inglés coronavirus disease 2019) es la enfermedad infecciosa causada por el virus SARS-CoV-2 (del inglés, severe acute respiratory syndrome coronavirus 2), de la familia de los coronavirus (Coronaviridae). Tanto este virus como la enfermedad que provoca eran desconocidos hasta que se desencadenara el brote asociado al mercado de alimentos de Wuhan (China) en diciembre de 2019. La epidemia de COVID-19 fue declarada emergencia de salud pública de preocupación internacional el 30 de enero de 2020 por la Organización Mundial de la Salud (OMS) y el 11 de marzo de 2020 fue caracterizada como una pandemia, al extenderse por todo el mundo ${ }^{i}$. En la actualidad ha afectado a 188 países, se han confirmado más de veintiún millones de casos y han fallecido 776.819 personas ${ }^{\mathrm{ii}}$, con consecuencias no sólo para la salud público sino también con profundos efectos sociales, económicos y ambientales. El epicentro de la pandemia se ha desplazado de Asia a Europa primeramente y posteriormente a América.

En lo que va del presente siglo, se han producido sucesivos brotes como el del SARS (síndrome respiratorio agudo grave o severo) en 2002-2004, a raíz de un coronavirus no conocido anteriormente en humanos, el de influenza A H5N1 (gripe aviar) en 2004-2006, la pandemia de gripe A H1N1 de 2009-2010, los brotes de la enfermedad del virus del Ébola, de los cuales el de 2014-2016 fue el más virulento y el de Zika en 2015-2016. Estos brotes han generado alerta en todo el planeta y han puesto en el debate público los criterios éticos para la investigación, vigilancia y atención de los pacientes en tales situaciones.

Es que toda pandemia, como problema de salud pública que trasciende los límites territoriales de los distintos países a los que afecta, debe ser abordada de manera integral e interdisciplinaria, pues excede el campo propio de la medicina, requiriéndose el aporte de varias disciplinas debido al abanico de consecuencias que produce ${ }^{\mathrm{iii}}$.

La primera y más importante obligación en situaciones de desastres naturales (tales como terremotos, tsunamis), conflictos bélicos y brotes de enfermedades es responder a las necesidades de salud de los individuos y las poblaciones, siendo la investigación biomédica parte de dicha respuesta.

Existe pues una obligación moral de poder aprender tanto como sea posible y de la forma más rápida para estar mejor preparados y, en brotes de enfermedades infecciosas como la COVID-19, tal obligación se traduce en una considerable presión para realizar investigaciones, 
por distintos motivos (su elevada tasa de contagio y/o mortalidad, las limitadas opciones de tratamiento y la ausencia de vacunas, etc.).

Ahora bien, estas investigaciones plantean desafíos trascendentes, pues suponen una tensión entre la necesidad de generar conocimiento rápidamente, mantener la confianza pública y superar obstáculos prácticos por una parte, y la obligación de asegurar la validez científica de las prácticas investigativas y el cumplimiento de todos los principios éticos por otra, procurando en todo momento no afectar la atención de la salud de manera indebida.

En lo que refiere a los modelos en investigación con seres humanos, el diseño de ensayos controlados aleatorizados se considera a menudo el "estándar por excelencia" para recolectar datos sólidos. Sin embargo, los requerimientos impuestos por la pandemia, permiten explorar diseños alternativos que puedan aumentar su eficiencia y el acceso a intervenciones experimentales prometedoras, siendo exigibles altos estándares éticos y la validez científica de sus resultados.

Asimismo, el contexto que supone la pandemia ha llamado a la reflexión ${ }^{\text {iv }}$ sobre las consideraciones metodológicas y éticas de los ensayos de desafío en humanos (Human challenge trials), en los cuales personas sanas voluntariamente son expuestas de manera intencional a un microorganismo infeccioso (OMS, 2016; OMS, 2020b). En los así denominados estudios controlados de infección humana (controlled human infection studies CHIs) un pequeño número de participantes está expuesto deliberadamente al patógeno para estudiar la infección y recopilar datos preliminares de eficacia de vacunas o tratamientos experimentales (JAMROZIK y SELGELID, 2020; SHAH et al., 2020).

Pues bien, luego de efectuar un breve e imprescindible recorrido por los principales documentos de nivel internacional referidos a la investigación con seres humanos en el contexto de emergencias sanitarias y comentar acerca del desarrollo de algunas investigaciones para responder a la actual pandemia, el presente artículo tiene la intención de presentar y reflexionar acerca de la respuesta que se dio sobre la temática en la Argentina, en particular, mediante la Resolución N 908/20 del Ministerio de Salud de la Nación.

\section{Normativa regulatoria de la investigación durante brotes de enfermedades}

La historia muestra innumerables ejemplos de abusos cometidos en la práctica de la investigación con seres humanos, por lo que a medida que los mismos fueron conocidos, existió

Revista RBBA Revista Binacional Brasil Argentina 
la preocupación por emitir normas protectorias de los sujetos que participan de dichas pesquisas.

Es así que se puede rastrear un hilo conductor de progreso moral que partió de considerar a la dignidad como cualidad inescindible de toda personav ${ }^{\mathrm{v}}$. Como consecuencia de ello, y en lo relativo a su participación en investigaciones biomédicas, es universalmente aceptado, entre otras pautas, que no debe realizarse experimento alguno sin el consentimiento voluntario de la persona ni cuando hay una razón a priori para suponer que puede ocurrir la muerte o una lesión irreparable $\mathrm{e}^{\mathrm{vi}}$. Asimismo, hay un consenso generalizado respecto a que los derechos e intereses de la persona que participa en la investigación tienen primacía por sobre todo otro interés, sea éste social o científico, tal como lo establecen la Declaración de Helsinski de la Asociación Médica Mundial de 1964 y modifs., el Convenio sobre Derechos Humanos y Biomedicina o Convenio de Oviedo de 1997 y la Declaración Universal de Bioética y Derechos Humanos de la UNESCO (2005), sólo por citar algunas ${ }^{\mathrm{vii}}$.

No obstante, haciendo un recorrido sobre los documentos internacionales clásicos vinculados con la investigación -comprendiendo a los citados, pero dejando fuera a las Pautas del Consejo de Organizaciones Internacionales de las Ciencias Médicas (CIOMS), a las que nos referiremos seguidamente-, se advierte que no contienen entre sus términos especificaciones para la regulación del desarrollo de investigaciones en situaciones de emergencias sanitarias o brotes de enfermedades. Lo mismo ocurre en documentos dedicados a la investigación con seres humanos emanados de prestigiosas organizaciones (Pautas y orientación operativa para la revisión ética de la investigación en salud con seres humanos, OMS, 2011; OPS, 2012) viii $^{\text {. }}$

En tanto, de las sucesivas versiones de las Pautas CIOMS, publicadas en 1982, 1993, 2002 y 2016, recién la última -denominada "Pautas éticas internacionales para la investigación relacionada con la salud con seres humanos" "ix - incluye una específica referida a la investigación en situaciones de desastre y brotes de enfermedades (pauta 20), que comentaremos sucintamente a continuación.

Tras considerar que las investigaciones relacionadas con la salud deberían formar parte de la respuesta a dichos eventos, y de destacar lo desafiante de la tarea investigativa en tales contextos, la citada pauta 20 puntualiza ciertos recaudos que investigadores, patrocinadores, etc. deberían asegurar que se cumplan. En una primera instancia, señala que aún en condiciones de emergencia, los estudios deben diseñarse de forma tal de producir resultados científicamente 
válidos. Refiere a que la investigación en situaciones de desastre debería planificarse con antelación, y resalta el deber de formular procedimientos para asegurar la existencia de mecanismos y pautas flexibles, convenientes y apropiados para la revisión y la supervisión éticas. Apunta que ello puede implicar, entre otras cosas, el presentar protocolos parciales del estudio para una "preevaluación" ética y la redacción de arreglos para la recolección y el intercambio de datos y muestras entre los colaboradores, lo cual no sustituye la revisión ética final de los protocolos específicos en un desastre.

Entre los comentarios, también sugiere que los comités de ética de la investigación o un comité de ética de especialistas (quizás de nivel nacional o regional) pueden realizar una revisión acelerada inicial de los protocolos del estudio y continuar la supervisión si tales estudios generan preocupaciones de índole ética importantes. La revisión ética local debería llevarse a cabo siempre que sea posible.

La misma pauta 20 en análisis exige asegurar: "que la investigación responde a las necesidades de salud o las prioridades de las víctimas del desastre y las comunidades afectadas y no puede realizarse fuera de una situación de desastre".

Asimismo, agrega como exigencia: "que los participantes se seleccionan de forma justa y se proporciona una justificación adecuada cuando se escogen o excluyen determinadas poblaciones", siendo aceptable dar prioridad al reclutamiento en un estudio a ciertos segmentos de la población; por ejemplo, los trabajadores de primera línea.

Tampoco excluye esta pauta la referencia a los demás recaudos éticos habituales de toda investigación en salud con seres humanos tales como: distribución equitativa de cargas y beneficios, evaluación realista de los riesgos y posibles beneficios individuales de intervenciones experimentales, participación activa de las comunidades en la planificación del estudio y obtención de consentimiento informado individual de los participantes -salvo situaciones de dispensa que se encuentan previstas en el mismo instrumento-.

Finalmente la pauta examinada exige la diseminación de los datos y resultados de la investigación como así también poner a disposición de las comunidades cualquier intervención efectiva desarrollada o conocimiento generado.

Más allá de los instrumentos internacionales recién citados, cabe destacar otros documentos producidos en el último quindenio que sirven de referencia a la hora de abordar la actual pandemia, tales como: International Health Regulations o IHR (OMS, 2005) ${ }^{\mathrm{x}}$; Ética de la investigación en la respuesta epidémica internacional: consulta técnica de la OMS $(2010)^{\mathrm{xi}}$; 
Ética en epidemias, emergencias y desastres: investigación, vigilancia y atención al paciente (OMS, 2015) ${ }^{\mathrm{xii}}$; Infecciones epidémicas emergentes y tratamientos médicos experimentales.

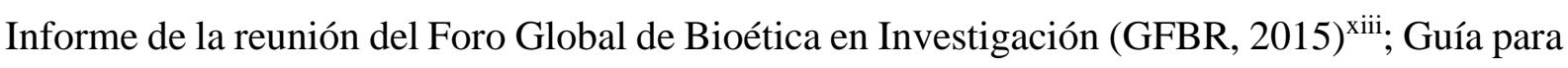
gestionar cuestiones éticas en brotes de enfermedades infecciosas (OMS, 2016) ${ }^{\mathrm{xiv}}$; Consulta de ética sobre el zika: Orientación ética sobre cuestiones clave planteadas por el brote (OMS, 2016 ${ }^{\mathrm{x} v}$ e Integrando la Investigación Clínica en la Respuesta Epidémica. La experiencia del ébola (National Academies of Sciences, Engineering and Medicine, 2017) ${ }^{\mathrm{xvi}}$.

Por su parte, desde la aparición de la COVID-19, resulta relevante destacar los siguientes documentos publicados: Investigación en emergencias sanitarias mundiales: cuestiones éticas (Nuffield Council on Bioethics, 2020) ${ }^{\text {xii }}$, Estándares éticos para la investigación durante emergencias de salud pública: destilando la orientación existente para apoyar la I+D de COVID-19 $9^{\mathrm{xviii}}$ (OMS, 2020a), Orientación para los comités de ética de investigación para la revisión rápida de la investigación durante emergencias de salud pública $(\mathrm{OMS}, 2020 \mathrm{c})^{\mathrm{xix}}$ y Orientación ética sobre cuestiones planteadas por la pandemia del nuevo coronavirus COVID-19 (OPS, 2020) ${ }^{\mathrm{xx}}$.

Asimismo, diferentes países del mundo han elaborado normativas específicas vinculadas a aspectos éticos relacionados con la COVID-19, algunas de alcance similar a la que analizaremos para el contexto $\operatorname{argentino}^{\mathrm{xxi}}$.

\section{Investigaciones actuales a nivel global}

El modelo imperante de Salud Basada en Evidencia tiene como objetivo poder aplicar de manera adecuada los resultados de la investigación científica a la práctica médica, con la finalidad de mejorar su efectividad y calidad. Uno de sus pilares es sin dudas la calidad de las investigaciones. En relación con la COVID-19 específicamente, en la base ClinicalTrials. gov ${ }^{\mathrm{xxii}}$, pueden identificarse 3017 estudios, de los cuales 1685 son ensayos clínicos, 28 estudios de fase 1 temprana (0,9\%), 200 de fase 1 (6,6\%), 660 de fase $2(21,9 \%)$, 390 de fase $3(13 \%)$ y 81 de fase 4 (2,7 \%). De ellos, 191 estudios (145 ensayos clínicos) corresponden a vacunas.

Un editorial de la prestigiosa revista científica Nature de fecha 14 de mayo ${ }^{\text {xxiii }}$ indica que la urgencia de encontrar tratamientos para la COVID-19 ha dado lugar a demasiados ensayos clínicos pequeños, que no emplean un número suficiente de sujetos de investigación 
para proporcionar resultados estadísticamente significativos y que no siempre incluyen un control. En tal sentido, el citado editorial considera conveniente el desarrollo de ensayos más grandes y más colaborativos. Refiere por su parte a las consecuencias de los diseños de los estudios de dos grupos de medicamentos: el remdesivir por un lado y la cloroquina e hidroxicloroquina por el otro.

El remdesivir se ha considerado como uno de los mejores candidatos para el tratamiento antiviral para el SARS-CoV-2. En un análisis temprano de 53 sujetos gravemente enfermos con COVID-19 en los Estados Unidos, Europa, Canadá y Japón a quienes se les dio el fármaco, el sesenta y ocho por ciento mostró una mejoría clínica (GREIN et al., 2020). Sin embargo, el estudio carecía de un grupo de control y no era un ensayo clínico organizado. Por el contrario, un ensayo aleatorizado controlado con placebo realizado en China que comenzó con 236 pacientes adultos hospitalizados no encontró beneficio significativo y propuso la realización de estudios más amplios (WANG et al., 2020). El 22 de mayo se publican los resultados de un ensayo doble ciego, aleatorizado, controlado con placebo con 1063 sujetos de investigación. Aquellos que recibieron remdesivir tuvieron un tiempo de recuperación promedio de 11 días en comparación con 15 días en aquellos que recibieron placebo, y las estimaciones de mortalidad a los 14 días fueron del 7,1\% con remdesivir y del 11,9\% con placebo (BEIGEL et al., 2020).

Por su parte, la hidroxicloroquina y la cloroquina, fármacos ya empleados en el tratamiento de la malaria y de enfermedades autoinmunes tales como artritis reumatoide o lupus eritematoso sistémico, proporcionan otro estudio de caso sobre las particularidades de las investigaciones en brotes de enfermedades. Después de que los primeros estudios en células cultivadas en laboratorio (estudio pre-clínico) sugirieran que los medicamentos podrían ser efectivos contra SARS-CoV-2 (WANG et al.; 2020), se lanzaron ensayos clínicos en todo el mundo, muchos de ellos pequeños y no controlados. Antes de contar con la evidencia suficiente sobre si las drogas eran efectivas y seguras, y a pesar de sus conocidos efectos citotóxicos sobre diferentes sistemas y órganos como el corazón, el 24 de abril el presidente de los Estados Unidos, Donald Trump, se apresuró a impulsar el tratamiento con hidroxicloroquina y cloroquina $^{\text {xxiv }}$. Prácticamente un mes después, el 22 de mayo, se publicó un estudio liderado por Mandeep Mehra, un conocido cirujano afiliado a la Universidad de Harvard, referido a un análisis multinacional del uso de hidroxicloroquina o cloroquina (con o sin un antibiótico macrólido), de tipo observacional -no un ensayo clínico-. La publicación incluyó datos de 671 hospitales de seis continentes (96.032 pacientes, 14.888 en los grupos de tratamiento y 81.144 
en el grupo de control) y profundizó aspectos de eficacia y seguridad en términos de mortalidad hospitalaria y aparición de arritmias ventriculares de novo, respectivamente (MEHRA et al., 2020). Todos los tratamientos experimentales mostraron mayor riesgo de mortalidad hospitalaria que el grupo control y también mayor riesgo cardíaco durante la hospitalización.

Finalmente, en fecha 5/6/2020, tres de los cuatro autores del artículo recién citado publicaron una retractación ${ }^{\mathrm{xxv}}$ y solicitaron que se lo retire, debido a preocupaciones con respecto a la veracidad de los datos y análisis realizados por Surgisphere Corporation y su fundador y coautor de la publicación original Sapan Desai. La empresa no había transferido toda la información necesaria para una adecuada revisión por pares ${ }^{x x v i}$. C. Chaccour, un investigador del Instituto de Salud Global de Barcelona, manifestó que "las dos revistas médicasmás prestigiosas nos han fallado", ya que deberían haber examinado más detenidamente la procedencia de los datos de Surgisphere antes de publicarlos ${ }^{\mathrm{xxvii}}$. Este caso trajo aparejados una llamada de atención sobre las publicaciones científicas y un cuestionamiento a la ciencia en un momento en que su contribución se ha vuelto de extrema necesidad para la salud global ${ }^{\mathrm{xxviii}}$.

Por su parte, esta situación recién descripta en forma sucinta tuvo sus consecuencias en el ensayo clínico internacional "Solidaridad", puesto en marcha por la OMS para la búsqueda de tratamientos eficaces y seguros contra la COVID-19. Su Grupo Ejecutivo en fecha 26 de mayo decidió implementar una pausa temporal del brazo de hidroxicloroquina como medida de precaución $^{\text {xxix }}$, mientras se revisaban los datos de seguridad. Aunque el 3 de junio la organización recomendó reanudarlos, el 17 de ese mes finalmente se anunció la interrupción del grupo de tratamiento con hidroxicloroquina ${ }^{\mathrm{xxx}}$.

\section{La cuestión en la República Argentina}

Es harto conocido el hecho de que en ciertos países latinoamericanos la temática relativa a la investigación biomédica se encuentra pobremente regulada, dominada por intereses privados (ANNAS, 2009), siendo éstos la mayoría de las veces de corporaciones trasnacionales, lo que da pie a situaciones propensas a la vulneración de los derechos de los participantes en las pesquisas. Argentina, sólo por lo que volcamos a continuación, puede considerarse entre estos países, independientemente de la evolución que ha tenido la actividad en las últimas dos 
décadas, acompañada por el cada vez más oído reclamo de bioeticistas para concientizar sobre la evaluación ética de las investigaciones con seres humanos.

Es que, no obstante la existencia de normas que unas pocas provincias poseen al respecto, no existe en el país un marco normativo adecuado, entendiendo por tal una ley emitida por el Congreso con alcance nacional que regule sistemáticamente toda investigación con seres humanos estableciendo los requisitos y procedimientos de modo de asegurar que sea respetuosa de la dignidad y de los derechos humanos de los participantes. Nobleza obliga: sí han existido planteos legislativos al respecto, pero sin mayor desarrollo.

Entre los antecedentes normativos nacionales sirve de fuente la "Guía para Investigaciones en Salud Humana", aprobada por la Resolución N 1480/11 del Ministerio de Salud de la Nación (B.O. 21/9/2011), y el "Régimen de Buena Práctica Clínica para Estudios de Farmacología Clínica”, estatuido por la Disposición 6677/10 de la Administración Nacional de Medicamentos, Alimentos y Tecnología Médica (ANMAT) (B.O. 05/11/2010) ${ }^{\text {xxxi }}$.

Por su parte, es de ineludible referencia la introducción del tema en nuestro derecho privado a partir del artículo 58 del Código Civil y Comercial de la Nación (aprobado por Ley $\mathrm{N}^{\circ}$ 26.944, B.O. 08/10/2014), que establece que: "La investigación médica en seres humanos mediante intervenciones, tales como tratamientos, métodos de prevención, pruebas diagnósticas o predictivas, cuya eficacia o seguridad no están comprobadas científicamente, sólo puede ser realizada si se cumple con los siguientes requisitos: (...)", fijando una serie de recaudos que el mismo artículo explicita en sus diez incisos. De ahí que hoy todo argentino puede conocer, a través del Código unificado, esa base mínima de requisitos que deben reunirse para justificar su participación en cualquier ensayo clínico que eventualmente se le proponga (CAPUTTO, 2017).

Ahora bien, más allá de tal observación, debemos admitir que sí representó un adelanto la creación en el año 2016 del Comité Nacional Asesor de Ética en Investigación, mediante Resolución del Ministerio de Salud de la Nación N ${ }^{\circ}$ 1002/16 (B.O. 21/7/2016) ${ }^{\text {xxxii }}$. Conformado por referentes de Comités Provinciales de Ética en Investigación, y distintas Comisiones Técnicas integradas por expertos, su razón de ser puede sintetizarse en dos puntos centrales: (i) entender en todo aspecto relacionado con las implicancias éticas de las investigaciones en salud en las que participen seres humanos, con el fin de proteger sus derechos fundamentales ponderando, a su vez, la necesidad de promover la investigación en salud; (ii) llevar adelante la acreditación de los Comités de Ética en Investigacion de los Institutos Nacionales y Revista RBBA $\mid$ Revista Binacional Brasil Argentina 
Organizaciones descentralizadas de la cartera sanitaria nacional (luego regulada por Res. $\mathrm{N}^{\circ}$ 2026/16, B.O. 18/11/2016).

Ya adentrándonos en la respuesta normativa argentina a la pandemia en cuanto refiere estrictamente a investigación biomédica, el 20 de marzo la ANMAT publicó una serie de medidas y recomendaciones en los estudios de farmacología clínica (EFC) ${ }^{\mathrm{xx} x i i i}$, expresando que el organismo priorizaría los estudios destinados a tratar o prevenir la enfermedad por coronavirus “(...) por lo que las evaluaciones se realizarán de manera acelerada conservando los más altos estándares para proteger la seguridad y los derechos de los participantes, así como la calidad de los datos".

Asimismo, la autoridad sanitaria, valiéndose del consejo del Comité de Expertos ${ }^{\mathrm{xxxiv}}$, el 17 de abril se manifestó conforme a la constitución de un Comité de Bioética para COVID-19 “(...) que elabore un documento con criterio ético y desde la perspectiva de derechos humanos, en el que la bioética acompañe las decisiones sanitarias basadas en evidencia científica durante la pandemia $(\ldots)^{9 \times x x v}$.

En esa misma fecha, por Resolución No 783/20 (B.O. 18/4/2020), el Ministro de Salud creó el "Plan Estratégico para regular el uso de plasma de pacientes recuperados de COVID-19 con fines terapéuticos", por el cual se buscó integrar todos los ensayos existentes en el país en lo que se denominó “Ensayo Clínico Nacional” (ECN), destinado a evaluar la seguridad y eficacia de dicha terapia.

Luego, por Resolución $\mathrm{N}^{\circ} 943 / 20$ (B.O. 21/5/2020), Argentina se alineó a la investigación sobre opciones terapeúticas no licenciadas para el tratamiento de los pacientes hospitalizados por COVID-19 promovida por la OMS, y conocida como Ensayo Clínico Aleatorizado "Solidaridad".

Asimismo, el portal ClinicalTrials. gov lista 33 estudios para COVID-19 en Argentina, 21 de los cuales son estudios intervencionales (ensayos clínicos) y 11 de carácter multicéntrico ${ }^{\mathrm{xxxvi}}$.

Por último, como se ha hecho público, recientemente el gobierno argentino anunció el acuerdo para la producción y distribución de la vacuna contra la COVID-19, por parte de empresas privadas del país y de México, con el fin de abastecer al mercado latinoamericano, excepto a Brasil, que tiene su propio acuerdo. Se trata del ensayo llevado adelante por la Universidad de Oxford y la farmacéutica mejicana AstraZeneca que se encuentra en fase 3, por lo que el proceso de producción se dará de manera simultánea a la espera de los resultados de 
aquél, como respuesta inaudita a la apremiante situación en que coloca la pandemia a los países de la región.

\section{Resolución $\mathbf{N}^{\circ}$ 908/20. Acotado análisis de su Anexo}

El 21 de abril, el Ministro de Salud de la Nación emitió la Resolución N 908/20 (B.O. 11/5/2020) mediante la cual se aprobraron las "Pautas Éticas y Operativas para la Evaluación Ética Acelerada de Investigaciones en Seres Humanos relacionadas con el COVID-19”xxxvii, metodólogicamente incluidas en el Anexo único indicado por su artículo 1ro.

El Anexo de la Resolución citada se divide en tres apartados: I. Introducción; II. Pautas para la evaluación; y III. Procedimientos operativos. Sobre este último no avanzaremos por cuestiones de extensión y por radicar el interés de este artículo fundamentalmente en el apartado que le precede.

La Introducción parte de reconocer que la pandemia nos enfrenta a mayores desafíos que los habituales por la necesidad de generar conocimiento rápidamente, y deja claro lo imperioso de armonizar dos caracteres de la evaluación ética: por un lado, su rigurosidad, en tanto las garantías éticas deben ser mayores en situaciones de emergencia y, por otro lado, su celeridad, requiriéndose adaptar los mecanismos habituales para lograr otros rápidos y flexibles en el nuevo contexto de la emergencia sanitaria.

Las Pautas para la Evaluación, dirigidas a los Comités de Ética de la Investigación $(\mathrm{CEIs})^{\mathrm{xxxviii}}$ se indican en diez incisos, los cuales transcribimos y, de manera sintética y críticamente, comentamos como sigue:

1.- La investigación no compromete la respuesta a la emergencia sanitaria generada por la pandemia. En particular la investigación no debe interferir indebidamente en la atención de las personas afectadas, ni en la labor del personal de salud ni en el funcionamiento del centro de salud en la que se realiza (p.1).

Las tareas desarrolladas en el marco de la investigación deben mantener indemnes las prestaciones sanitarias brindadas en función de la emergencia, teniendo presente que la prioridad estriba en la atención de la salud de los afectados. Pauta semejante a la que se puede encontrar en los documentos que le sirven de fuente ${ }^{\text {xxxix }}$, aquí se agrega la no interferencia en la labor del personal sanitario ni el funcionamiento del centro de salud involucrado. 
2.- La investigación debe tener valor social y ser relevante, es decir, responder a las necesidades de salud o las prioridades de las personas y las comunidades afectadas. El conocimiento que se busca obtener no debería poder ser generado por otros medios alternativos y la investigación no podría realizarse fuera de la situación de emergencia sanitaria (p.2).

A los fines de evaluar estos indicadores de la investigación sobre la COVID-19, consideramos adecuada las recomendaciones respecto a involucrar a la comunidad antes de iniciar una investigación para garantizar que los estudios sean sensibles a las necesidades y las prioridades locales, que el diseño del estudio sea aceptable para la población donde se llevará a cabo y que los resultados les sean accesibles ${ }^{\mathrm{xl}}$.

También expresa la premisa básica para la justificación ética de la investigación con seres humanos, cual es que no existan medios alternativos a la participación de personas para la generación del conocimiento, agregándose la condición de impostergabilidad.

Aún así, consideramos mejorable su redacción, debiendo leerse al inicio: "la investigación tenga valor social y sea relevante”.

3.- Los estudios están diseñados de forma de producir resultados científicamente válidos. Dada la necesidad de producir evidencia válida rápidamente pueden presentarse propuestas de diseños alternativos de ensayos clínicos. Se requiere evaluar cuidadosamente su validez científica y no confundir diseños alternativos, con diseños científicamente inválidos o poco sólidos (p.2).

Replicando una máxima ética fundamental en materia de investigación biomédica, este ítem refiere a que los diseños de los ensayos, aún los alternativos motivados en su necesaria y conveniente aceleración, se presenten de manera tal que confluyan en resultados científicos válidos. Este inciso se halla en línea con lo publicado por la OMS (2020a), que establece debe evitarse la ortodoxia metodológica (por ejemplo, en favor de un diseño de ensayo particular) para considerar críticamente la investigación y poder responder preguntas de investigación específicas con datos rigurosos y confiables para garantizar que los resultados sean válidos y útiles.

4.- Los participantes se seleccionan de forma justa cuando se prioriza a determinado grupo sobre otro y se proporciona una justificación pública y transparente adecuada para dicha priorización.

5.- La incertidumbre, los riesgos y posibles beneficios individuales de intervenciones experimentales se evalúan de manera realista considerando la evidencia existente, especialmente cuando se encuentran en las primeras etapas de desarrollo (p. 2). 
Con respecto al inciso 4, entendemos preferible la siguiente redacción: "los participantes se seleccionan de manera justa, debiéndose proporcionar una justificación pública y transparente cuando se priorice a determinado grupo sobre otro".

En principio, los participantes deben ser tratados con igual respeto. La selección de los participantes depende de los objetivos y del diseño del estudio, y de la mayor probabilidad de minimizar los riesgos y maximizar los beneficios a nivel individual, pudiendo justificarse la exclusión de grupos o individuos cuando exista una razón científica o de seguridad debidamente fundamentada ${ }^{x l i}$. Así, grupos considerados "vulnerables", tales como mujeres embarazadas, minorías, niños, entre otros no deben ser excluidos rutinariamente de la participación en la investigación sin esta razonable justificación.

En cuanto al inciso 5, a la clásica balanza riesgos/beneficios se agrega ahora la incertidumbre propia de lo que estamos enfrentando, incertidumbre que vuelve necesaria la vigilancia en pos de reducirla en la medida de lo posible, y que justifica que las medidas que se toman en un momento puedan ser modificadas de acuerdo a la dinámica de la emergencia sanitaria (ANMAT, 2020; OPS, 2020).

6.- Se presenta un plan de minimización de los riesgos por la emergencia sanitaria para los participantes, investigadores y los profesionales de la salud que realizan investigaciones en el contexto de la pandemia para extremar las medidas tendientes a evitar el contagio y diseminación de COVID-19 así como la saturación del sistema de salud (p.2).

La presentación de ensayos vinculados a la COVID-19 debe ir acompañada de un plan o programa que evidencie las medidas de seguridad y prevención que se habrán de tomar respecto a todos los intervinientes en la investigación, antes y durante su desarrollo.

7.- Se obtiene el consentimiento informado individual de los participantes o sus representantes legales, apoyo, familiares o allegados, a menos que se cumplan las condiciones para las excepciones del consentimiento informado. Acorde a la Ley de Derechos del Paciente $\mathrm{N}^{\circ} 26.529$ las excepciones son: a) Cuando mediare grave peligro para la salud pública; b) Cuando mediare una situación de emergencia, con grave peligro para la salud o vida del paciente, y no pudiera dar el consentimiento por sí o a través de sus representantes legales. Asimismo, conforme el Decreto No 1089/2012 las excepciones deben ser interpretadas con carácter restrictivo y asentadas en la historia clínica del paciente, fundamentando los motivos por los cuales no pudo obtenerse y las medidas adoptadas.

8.- El proceso de consentimiento se diseña acorde a la situación de los afectados y se enfatiza en la diferencia entre atención de la salud e 
investigación para que la persona decida libremente. Es muy probable que los mecanismos habituales de toma de consentimiento no sean óptimas, por lo que será necesario considerar formas alternativas. En estos casos el profesional tratante, deberá asentar en la historia clínica la fecha y alcance de cómo se realizó el proceso de consentimiento y su fundamento.

9.- Se promueve la obtención del consentimiento amplio para usos futuros de las muestras y datos de los participantes y pacientes afectados, en investigaciones que tengan como objetivo dar respuesta a la pandemia. Es recomendable obtener el consentimiento informado para investigaciones futuras desde el momento en que se diagnostica al paciente (p. 2-3).

Aclaramos que la cuestión relativa al consentimiento informado es tan compleja que excede el marco del presente trabajo, no obstante lo cual ponemos de relieve algunas inquietudes que despiertan estos incisos.

En primer lugar, se alude a la Ley de Derechos del Paciente $N^{\circ} 26.259$ (B.O. 20/11/2009) y su decreto reglamentario $N^{\circ}$ 1089/2012 (B.O. 05/7/2012), normativa que, si bien menciona en varias oportunidades a la investigación, lo hace en términos generales, no contando con un tratamiento adecuado del consentimiento informado aplicable a ensayos clínicos.

Llama la atención que no se haga referencia a los arts. 58 y 59 del Código Civil y Comercial de la Nación que tratan sobre Investigaciones en Seres Humanos y Consentimiento Informado para actos médicos e investigaciones en salud, respectivamente, contemplando el eventual caso en que los ensayos incluyan a voluntarios sanos, no pacientes en sentido estricto. Se sabe que el consentimiento informado en investigación contiene especificaciones diversas respecto al de la práctica clínica, que tampoco fueron abordadas en el art. 59 del Código unificado, sí en actos administrativos de inferior jerarquía ya citados.

En consecuencia, consideramos que la Resolución $N^{\circ}$ 1840/11 y la Disposición de la ANMAT 6677/10 resultan de referencia más compatible y se ajustan a las indicadas por las pautas éticas internacionales, por ejemplo, estableciendo otras excepciones a la obtención del consentimiento informado o a requisitos específicos del proceso.

En segundo lugar, la primera parte del inciso 8 entendemos responde a la necesidad de que la toma del consentimiento informado -correctamente indicada como proceso- se ajuste a las condiciones de competencia y posibilidades concretas de toma de decisión de los participantes. A tales efectos, será preciso considerar su estado de salud, y eliminar en cuanto sea posible las barreras de toda índole para el consentimiento informado, no sólo las culturales, 
lingüísticas, entre otras, sino las que se relacionen con el miedo y la desesperación que supone vivir la pandemia.

Amén de lo expuesto, y teniendo en cuenta que en crisis humanitarias puede resultar muy difícil para los participantes distinguir entre las actividades de investigación y la respuesta sanitaria, especialmente cuando están asociadas al cuidado de la salud ${ }^{x l i i}$, se resalta que las personas siempre deben saber si están recibiendo atención médica y si la misma involucra también la participación en un ensayo o intervención de otra naturaleza.

Ahora, relacionado con el comentario anterior, sólo parecería referir a investigaciones sobre pacientes, en cuanto alude a "historia clínica" y a "médico tratante"; no obstante, nuevamente creemos que debió preveerse y distinguirse claramente la situación del sujeto de investigación que es, a su vez, paciente, y el individuo voluntario sano que se presta a la investigación. Ergo, sólo tratándose de pacientes corresponde tomar registro en la historia clínica.

El inciso 9, por su parte, trata sobre los usos futuros de las muestras biológicas de los participantes. Dos observaciones: (i) al referir al objetivo de las investigaciones, relacionado con la pandemia, prevé su uso futuro directo, y no secundario; (ii) recomienda como pauta temporal la obtención temprana del consentimiento informado, al ser diagnosticado el paciente, dejando fuera nuevamente a los eventuales participantes sanos.

Finalmente, el apartado II, expresa en su inciso 10: “ Los resultados de la investigación se diseminan, los datos se comparten y cualquier intervención efectiva desarrollada o conocimiento generado se pone a disposición de las comunidades afectadas".

Es preciso tener en cuenta que en la investigación desarrollada en el marco de la pandemia no sólo se acelera la revisión ética del protocolo, sino que el acortamiento de los tiempos comprende también a la publicación de los resultados, incluidos -por honestidad científica- tanto los positivos como los negativos, por lo que la evaluación de su seriedad y solvencia se torna aún más exigente de lo habitual.

Es definitivamente un tema bioético el relativo a los sesgos en la diseminación de los resultados a través de distintas metodologías como la publicación repetida, minimización o enmascaramiento de los efectos secundarios, la maximización de tendencias que no son estadísticamente significativas (PERALTA, 2005), la falsificación, el “dibujo" (fabrication) de datos y el plagio (CECTE, 2005). 
De acuerdo con lo detallado en el apartado 3 de este trabajo, la pandemia ha generado una presión a los procesos estandarizados de publicación de las revistas científicas para acelerar la aparición de las contribuciones. En este sentido, los respositorios de prepublicaciones o preimpresiones (preprints) permiten la colocación y difusión de manuscritos sin revisión por pares (peer review), edición o control de fraude.

Allende lo expuesto, destacamos que el apartado que estamos analizando no prevé nada tocante a la privacidad del sujeto y a la confidencialidad de los datos colectados, no obstante lo cual debe interpretarse que corresponde sean respetadas por tratarse de un requisito inherente a toda investigación, considerada incluso un principio para algunos documentos ${ }^{\text {xliii }}$.

Entendemos que lo estipulado en relación con la disposición a favor de las comunidades afectadas de las intervenciones efectivas o conocimientos generados busca evitar las inequidades tan frecuentes en la actividad, lo que se enfrenta con la cuestión de las patentes en el complejo médico industrial. En este sentido, consideramos oportuno resaltar la importancia del denominado “Acceso Mancomunado a Tecnología contra la COVID-19” (COVID-19 Technology Access Pool, o C-TAP) ${ }^{\text {xliv }}$ de la OMS, que invita a las empresas o gobiernos que desarrollen un tratamiento eficaz a ceder la patente al Banco de Patentes de Medicamentos, que posteriormente concederá una sublicencia a los fabricantes de medicamentos genéricos para su explotación. La OMS reconoce la importancia de las patentes para estimular la innovación, pero considera que las personas deben ser la prioridad, siendo las tecnologías para prevenir, detectar y tratar la COVID-19 un bien público mundial al que todas las personas deben poder acceder.

\section{Conclusiones}

Hemos intentado reflexionar sobre algunas de las inquietudes que despierta la actividad investigativa con seres humanos frente al actual panorama, signado por la pandemia de la COVID-19. Hoy más que nunca, resulta imperioso el auxilio de la ciencia a fin de sortear este mal trance para la humanidad cumpliendo con la normativa existente, respetuosa -a su vez- de la dignidad de la persona y de sus derechos humanos. Ahora, conforme enseña la historia de la investigación relacionada con la salud humana, no cualquier esfuerzo se encontrará científica y éticamente validado, sino sólo aquél que propenda al conocimiento generalizable y que dé cumplimiento a las pautas de las que hemos hecho mención, pensadas para la tutela de los participantes en los ensayos y que han sido elaboradas por distintos organismos y países.

Revista RBBA $\mid$ Revista Binacional Brasil Argentina 
En tal camino, a nuestro entender, la respuesta de Argentina a la problemática de la investigación en el marco de la emergencia sanitaria agravada por la pandemia viene siendo elogiable, por su oportunidad, por el hecho de haber sido dispuesta pese a las deficiencias normativas históricas de las que hicimos referencia muy someramente, y por su notoria búsqueda de proteger la integridad y la primacía de los derechos e intereses de los participantes en los ensayos.

\section{REFERENCIAS}

ANMAT. Medidas y recomendaciones en los estudios de farmacología clínica durante la pandemia COVID19, 2020 [en línea]. Disponible en: https://www.argentina.gob.ar/noticias/medidas-y-recomendaciones-en-los-estudios-defarmacologia-clinica-durante-la-pandemia-covid [Consulta: 16 junio 2020].

ANNAS, G.J. Globalized Clinical Trials and Informed Consent. The New England Journal of Medicine, v. 360, p. 2050-2053, 2009. [en línea]. Disponible en: https://www.nejm.org/doi/full/10.1056/nejmp0901474 [Consulta: 15 junio 2020].

BEIGEL, J.H., TOMASHEK, K.M., DODD, L.E., MEHTA, A.K., ZINGMAN, B.S., KALIL, A. C. LANE, H.C. Remdesivir for the Treatment of Covid-19 -Preliminary Report. The New England Journal of Medicine, p. 1-12, 2020. [en línea]. Disponible en: https://www.nejm.org/doi/pdf/10.1056/NEJMoa2007764?articleTools=true [Consulta: 15 junio 2020].

CAPUTTO, M.C. La investigación con seres humanos: una novedosa y saludable introducción del artículo 58 del Código Civil y Comercial de la Nación. Revista La Ley, ADLA v. 10, 59, 2017.

COMITÉ NACIONAL DE ÉTICA EN LA CIENCIA Y LA TECNOLOGÍA (CECTE). Análisis y recomendaciones para una ética en las prácticas de la investigación, 2005 [en línea]. Disponible en: www.cecte.gov.ar/str/etica/_[Consulta: 15 junio 2020].

GREIN, J., OHMAGARI, N., SHIN, D., DIAZ, G., ASPERGES, E., CASTAGNA, A. FLANIGAN, T. Compassionate Use of Remdesivir for Patients with Severe Covid-19. The New England Journal of Medicine, v. 382, p. 2327-2336, 2020. [en línea]. Disponible en: https://www.nejm.org/doi/10.1056/NEJMoa2007016 [Consulta: 15 junio 2020].

JAMROZIK, E. Y SELGELID, M.J. COVID-19 human challenge studies: ethical issues. [en línea]. The Lancet Infectious Diseases, p. 1-6, 2020. [en línea]. Disponible en: https://www.thelancet.com/journals/laninf/article/PIIS1473-3099(20)30438-2/fulltext\#backbib8 [Consulta: 19 junio 2020].

LONDON, A.J. Y JONATHAN KIMMELMAN, J. Against pandemic research exceptionalism. Science, v. 368, n. 6490, p. 476-477, 2020. [en línea]. Disponible en: https://science.sciencemag.org/content/368/6490/476 [Consulta: 22 junio 2020]. 
MEHRA, M.R., DESAI, S.S., KUY, S.R., HENRY, T.D. Y PATEL, A.N. Cardiovascular Disease, Drug Therapy, and Mortality in Covid-19. The New England Journal of Medicine, 382: e102, p. 1-7, 2020. [en línea]. Disponible en: https://www.nejm.org/doi/pdf/10.1056/NEJMoa2007621 [Consulta: 19 junio 2020].

MEHRA, M.R., DESAI, S.S., RUSCHITZKA, F. Y PATEL, A.N. Hydroxychloroquine or chloroquine with or without a macrolide for treatment of COVID-19: a multinational registry analysis. The Lancet, p. 1-10, 2020. [en línea]. Disponible en: https://doi.org/10.1016/S01406736(20)31180-6 [Consulta: 22 junio 2020].

MEHRA, M.R., DESAI, S.S., KUY, S.R., HENRY, T.D. Y PATEL, A.N. Retraction: Cardiovascular Disease, Drug Therapy, and Mortality in Covid-19. N Engl J Med. DOI: 10.1056/NEJMoa2007621. The New England Journal of Medicine, v. 382, p.2582, 2020. [en línea]. Disponible en: https://www.nejm.org/doi/full/10.1056/NEJMc2021225 [Consulta: 19 junio 2020].

ORGANIZACIÓN PANAMERICANA DE LA SALUD. Orientación ética sobre cuestiones planteadas por la pandemia del nuevo coronavirus (COVID-19), 2020. [en línea]. Disponible en: https://www.paho.org/es/documentos/orientacion-etica-sobre-cuestiones-planteadas-porpandemia-nuevo-coronavirus-covid-19 [Consulta: 21 junio 2020].

PERALTA, V. Ensayos clínicos, industria farmacéutica y práctica clínica. Boletín de Información Farmacoterapéutica de Navarra, v. 13, n. 4, p. 30-34, 2005.

RUBIN, E.J. Expression of Concern: Mehra MR et al. Cardiovascular Disease, Drug Therapy, and Mortality in Covid-19. N Engl J Med. DOI: 10.1056/NEJMoa2007621 (Editorial). The New England Journal of Medicine, v. 382, p. 2464, 2020. [en línea]. Disponible en: https://www.nejm.org/doi/full/10.1056/NEJMe2020822 [Consulta: 19 junio 2020].

SHAH, S.K., MILLER, F.G., DARTON, T.C., DUENAS, D., EMERSON, C., FERNANDEZ LYNCH, H.,...RID, A. Ethics of controlled human infection to address COVID-19. Science, v. $368, \quad$ n. 6493 , p. 832-834, 2020. [en línea]. Disponible en: https://science.sciencemag.org/content/368/6493/832 [Consulta: 22 junio 2020].

TEALDI, J.C. (COORD.), PFEIFFER, M.L., SAVOY, A. Y BLANCO, L.G. Consulta Pública Para un Sistema Nacional de Ética y Derechos Humanos en las Investigaciones Biomédicas. Informe Final, 2006. [en línea]. Disponible en: https://www.psi.uba.ar/academica/carrerasdegrado/psicologia/sitios_catedras/obligatorias/723 _etica2/material/noticias/consulta_publica_senado_nacion.pdf [Consulta: 16 junio 2020].

WANG, M., CAO, R., ZHANG, L., YANG, X., LIU, J., XU, M.,..XIAO, G. Remdesivir and chloroquine effectively inhibit the recently emerged novel coronavirus (2019-nCoV) in vitro. Cell Res, v. 30, p. 269-271, 2020. [en línea]. Disponible en: https://doi.org/10.1038/s41422020-0282-0 [Consulta: 15 junio 2020].

WANG, Y., ZHANG, D., DU, G., DU, R., ZHAO, J., JIN, Y.,... WANG, C. Remdesivir in adults with severe COVID-19: a randomised, double-blind, placebo-controlled, multicentre trial. The Lancet, v. 395, p. 1569-78, 2020. [en línea]. Disponible en: 
https://www.thelancet.com/pdfs/journals/lancet/PIIS0140-6736(20)31022-9.pdf [Consulta: 15 junio 2020].

WORLD HEALTH ORGANIZATION. Human Challenge Trials for Vaccine Development: regulatory considerations, 2016. [en línea]. Disponible en: https://www.who.int/biologicals/expert_committee/Human_challenge_Trials_IK_final.pdf [Consulta: 19 junio 2020].

WORLD HEALTH ORGANIZATION. Ethical standards for research during public health emergencies: Distilling existing guidance to support COVID-19 R\&D, 2020a. [en línea]. Disponible en: https://www.who.int/publications/i/item/WHO-RFH-20.1 [Consulta: 21 junio 2020].

WORLD HEALTH ORGANIZATION. Key criteria for the ethical acceptability of COVID19 human challenge studies, 2020b. [en línea]. Disponible en: https://www.who.int/ethics/publications/key-criteria-ethical-acceptability-of-covid-19-humanchallenge/en/ [Consulta: 22 junio 2020].

WORLD HEALTH ORGANIZATION. Guidance for research ethics committees for rapid review of research during public health emergencies, 2020c. [en línea]. Disponible en: https://www.who.int/publications/i/item/9789240006218 [Consulta: 21 junio 2020].

\section{Notas}

\footnotetext{
${ }^{\mathrm{i}}$ https://www.paho.org/es/tag/enfermedad-por-coronavirus-covid-19_[Consulta: 1 junio 2020].

ii https://coronavirus.jhu.edu/map.html_[Consulta: 17 agosto 2020].

iii Además de los ensayos clínicos que evalúan diagnósticos, tratamientos o vacunas, se requieren investigaciones epidemiológicas, de las ciencias sociales y estudios de implementación de políticas o programas, que pueden jugar un papel crítico en la reducción de la morbilidad y mortalidad y abordar las consecuencias comunitarias y económicas causadas por el brote (London y Kimmelman, 2020).

iv https://www.thehastingscenter.org/human-challenge-studies-for-covid-19-vaccine-questions-about-benefitsand-risks/?fbclid=IwAR2gLV4ENr9IRHA3jIRCdZ70R6q_duquEKLOlwxfOvyX8Vj4SRbTmWaRpPs

[Consulta: 19 junio 2020].

" La Declaración Universal de Derechos Humanos (ONU,1948) en su artículo 1 establece: "Todos los seres humanos nacen libres e iguales en dignidad (...)". https://www.un.org/es/universal-declaration-human-rights/ [Consulta: 20 junio 2020].

vi Código de Núremberg (1947). http://www.bioeticanet.info/documentos/Nuremberg.pdf_[Consulta: 20 junio 2020].

vii Se hace referencia específicamente al artículo 5 de la Declaración de Helsinki original (1964), hoy artículo 8 (versión de 2013), al Artículo 2 (Primacía del ser humano) del Convenio del Consejo de Europa para la protección de los derechos humanos y la dignidad del ser humano respecto de las aplicaciones de la biología y la medicina o Convenio de Oviedo (1997) y al artículo 3 de la Declaración Universal sobre Bioética y Derechos Humanos (2005).

https://www.wma.net/es/policies-post/declaracion-de-helsinki-de-la-amm-principios-eticos-para-las-

investigaciones-medicas-en-seres-humanos/._Consulta: 20 junio 2020].

https://www.coe.int/en/web/bioethics/oviedo-convention_[Consulta: 20 junio 2020].

https://unesdoc.unesco.org/ark:/48223/pf0000142825_spa.page=85_[Consulta: 20 junio 2020].

viii https://www.who.int/ethics/publications/9789241502948/en/_[Consulta: 20 junio 2020].

https://www.paho.org/hq/dmdocuments/2013/Etica-pautas2013.pdf_[Consulta: 20 junio 2020].

ix https://cioms.ch/wp-content/uploads/2017/12/CIOMS-EthicalGuideline_SP_INTERIOR-FINAL.pdf_Consulta: 20 junio 2020].

${ }^{\mathrm{x}}$ https://www.who.int/ihr/en/_[Consulta: 21 junio 2020].
} 
${ }^{\text {xi }}$ El encuentro se llevó a cabo en Ginebra, Suiza en junio de 2009 y fue publicado en 2010. https://apps.who.int/iris/handle/10665/70739 [Consulta: 21 junio 2020].

xii https://www.who.int/ethics/publications/epidemics-emergencies-research/en/ [Consulta: 21 junio 2020].

xiii http://www.gfbr.global/wp-content/uploads/2016/03/GFBR-2015-meeting-report-emerging-epidemicinfections-and-experimental-medical-treatments.pdf [Consulta: 21 junio 2020].

xiv https://apps.who.int/iris/handle/10665/250580_[Consulta: 21 junio 2020].

${ }^{x v}$ https://iris.paho.org/handle/10665.2/28485 [Consulta: 21 junio 2020].

xvi https://www.nap.edu/catalog/24739/integrating-clinical-research-into-epidemic-response-the-ebola-experience [Consulta: 21 junio 2020].

xvii Publicado en inglés el 28/1/2020. Disponible en: https://www.nuffieldbioethics.org/publications/research-inglobal-health-emergencies_[Consulta: 21 junio 2020].

xviii Publicado en inglés el 29/3/2020. Disponible en: https://www.who.int/publications/i/item/WHO-RFH-20.1 [Consulta: 21 junio 2020].

xix Publicado en inglés el 28/5/2020. Disponible en: https://www.who.int/publications/i/item/9789240006218 [Consulta: 21 junio 2020].

xx Publicado el 16/3/2020. Disponible en: https://www.paho.org/es/documentos/orientacion-etica-sobrecuestiones-planteadas-por-pandemia-nuevo-coronavirus-covid-19_[Consulta: 21 junio 2020].

${ }^{x \times i}$ https://www.who.int/ethics/topics/outbreaks-emergencies/covid-19/en/ [Consulta: 27 junio 2020].

xxii https://clinicaltrials.gov/_[Consulta: 17 agosto 2020].

xxiii https://media.nature.com/original/magazine-assets/d41586-020-01391-9/d41586-020-01391-9.pdf_[Consulta: 15 junio 2020].

xxiv https://www.washingtonpost.com/politics/2020/04/24/rise-fall-trumps-obsession-with-hydroxychloroquine/ [Consulta: 15 junio 2020].

xxv https://www.thelancet.com/journals/lancet/article/PIIS0140-6736(20)31324-6/fulltext\#bib1__Consulta: 15 junio 2020].

MEHRA et al. habían publicado otro artículo el 1/5/2020 en The New England Journal of Medicine, en el que sugerían que la enfermedad cardiovascular subyacente se asocia con un mayor riesgo de muerte entre los pacientes hospitalizados con Covid-19. En fecha 2/6 se publica un editorial (RUBIN, 2020) que plantea preocupaciones importantes sobre la calidad de la información en la base de datos que emplea esa investigación y solicitaron a los autores que aporten evidencia de que los datos son confiables. En fecha $4 / 6$ los autores solicitaron que se retire el artículo, debido a que no se les otorgó acceso a los datos en bruto y éstos no se pudieron poner a disposición de un auditor externo para su validación.

xxvi En redes como Twitter se conoce este caso como el escándalo del \#LancetGate.

xxvii https://www.sciencemag.org/news/2020/06/mysterious-company-s-coronavirus-papers-top-medical-journalsmay-be-unraveling [Consulta: 20 junio 2020].

xxviii https://www.agenciasinc.es/Opinion/El-escandalo-del-LancetGate-y-la-hidroxicloroquina-una-llamada-deatencion-sobre-las-publicaciones-cientificas [Consulta: 20 junio 2020].

xxix https://www.agenciasinc.es/Noticias/La-OMS-suspende-por-seguridad-los-ensayos-con-hidroxicloroquinaen-coronavirus [Consulta: 20 junio 2020].

xxxhttps://www.who.int/es/emergencies/diseases/novel-coronavirus-2019/global-research-on-novel-coronavirus2019-ncov/solidarity-clinical-trial-for-covid-19-treatments_Consulta: 26 junio 2020].

xxxi Ambos documentos se encuentran disponibles en:

http://www.msal.gob.ar/images/stories/bes/graficos/0000001274cnt-guia_investigaciones_salud_humana.pdf [Consulta: 16 junio 2020].

xxxii Disponible en: https://www.boletinoficial.gob.ar/pdf/linkQR/QUp2MjdZblBnZmRycmZ0RFhoUThyQT09. Podemos citar como interesante antecedente de un ente similar, el primero con incumbencia nacional, al Comité Nacional de Ética en la Ciencia y la Tecnología (CECTE), creado en abril de 2001 mediante Resolución N 4/2001 por la entonces Secretaría de Ciencia, Tecnología e Innovación Productiva.

xxxiii ANMAT. Medidas y recomendaciones en los estudios de farmacología clínica durante la pandemia COVID19, entre las cuales figura la comunicación a los participantes de las recomendaciones del Ministerio de Salud para prevenir el contagio y diseminación de la enfermedad, el encargo a los patrocinadores de los EFC de elaborar un plan de mitigación de riesgo con idénticos fines, la suspensión del reclutamiento -salvo para pesquisas relacionadas con la pandemia-, las medidas a adoptar en caso de resultar necesario realizar visitas presenciales, el acceso seguro al medicameno del estudio, etc. Todo ello para garantizar el bienestar y seguridad de los participantes. Disponible en: https://www.argentina.gob.ar/noticias/medidas-y-recomendaciones-en-los-estudiosde-farmacologia-clinica-durante-la-pandemia-covid. [Consulta: 16 junio 2020]. 
xxxiv Este comité, conformado por siete prestigiosos especialistas infectólogos y epidemiólogos de Argentina, en base a la vigilancia epidemiológica, viene aconsejando al Poder Ejecutivo en la toma de decisiones que hacen a lo relativo, vgr., al aislamiento social preventivo y obligatorio, sus sucesivas extensiones, fases y flexibilización.

xxxv https://www.argentina.gob.ar/noticias/salud-convoco-especialistas-en-bioetica-para-conformar-un-comitedurante-la-pandemia [Consulta: 31 mayo 2020].

xxxvi https://clinicaltrials.gov/ [Consulta: 17 agosto 2020].

xxxvii http://iah.salud.gob.ar/doc/471.pdf [Consulta: 31 mayo 2020].

xxxviii Este documento es anterior al publicado por la OMS el 28/5/2020 - "Guidance for research ethics committees for rapid review of research during public health emergencies"- Disponible en: https://www.who.int/publications/i/item/9789240006218 [Consulta: 28 junio 2020].

xxxix Vgr., Nuffield Council on Bioethics (Reino Unido), Research in global health emergencies: ethical issues. Short Report, p. 5, que enfáticamente condiciona el respaldo a la investigación a que se aborden las necesidades sanitarias básicas de quienes participan en la misma a través del esfuerzo de respuesta, recomendando incluso no invitar a participar en investigaciones biomédicas cuando sus necesidades básicas no están satisfechas. Disponible en: www.nuffieldbioethics.org [Consulta: 16 junio 2020].

${ }^{x l}$ De hecho, el primer desafío ético del informe de Nuffield refiere a cuestiones de poder e influencia, indagándose cómo se incluyen las voces de los más afectados por la emergencia en las decisiones sobre qué, dónde y cómo se investiga.

xli Cfr. Res. 1480/11, apartado A5.

xlii Cfr. Nuffield Council on Bioethics.

xliii Vgr., en la Declaración Universal de Bioética y Derechos Humanos, UNESCO, 2005. Artículo 9. http://portal.unesco.org/es/ev.php-URL_ID=31058\&URL_DO=DO_TOPIC\&URL_SECTION=201.html [Consulta: 15 junio 2020].

${ }^{\text {xliv }}$ https://www.who.int/emergencies/diseases/novel-coronavirus-2019/global-research-on-novel-coronavirus2019-ncov/covid-19-technology-access-pool_Consulta: 28 junio 2020].

El C-TAP tiene cinco prioridades:

- fomentar la divulgación pública de los estudios sobre la secuencia genética del virus.

- promover la divulgación pública de todos los resultados de ensayos clínicos.

- alentar a los gobiernos y otras entidades que financian investigaciones a incluir disposiciones en los contratos con las empresas farmacéuticas sobre la diseminación y la publicación equitativas de datos sobre ensayos clínicos. - conceder autorizaciones a grandes y pequeños fabricantes para producir tratamientos y vacunas.

- promover modelos abiertos de innovación y transferencia tecnológica para aumentar la capacidad local de fabricación y suministro.

https://www.who.int/es/dg/speeches/detail/who-director-general-s-opening-remarks-at-the-media-briefing-oncovid-19---29-may-2020 [Consulta: 28 junio 2020]. 